\title{
General Directionality and the Local Behavior of Argumentation Semantics ${ }^{\star}$
}

\author{
Cristian Gratie $^{1}$, Adina Magda Florea ${ }^{1}$, and John-Jules Ch. Meyer ${ }^{2}$ \\ 1 AI-MAS Laboratory, Computer Science Department \\ University "Politehnica" of Bucharest, Romania \\ E-mail: cristian.gratie@cs.pub.ro, adina.florea@cs.pub.ro \\ 2 Intelligent Systems Group, Computer Science Department \\ Utrecht University, The Netherlands \\ E-mail: J.J.C.Meyer@uu.nl
}

\begin{abstract}
In abstract argumentation, the directionality principle conveys the intuition that, for an unattacked set, the choice of arguments that are part of an extension should only depend on the restriction of the framework to that set. Furthermore, having made such a choice, one should be able to select arguments from the rest of the framework so as to get an extension. In this paper we show how this idea can be generalized and used for formulating SCC-recursiveness as a stronger version of directionality.

We argue that such properties characterize the information that is needed for computing the extensions of an argumentation semantics. We provide a formal approach for describing and comparing directionality-like properties. Our model provides a clear distinction between SCC-recursive semantics that use defense information and those that do not use it.
\end{abstract}

Keywords: argumentation frameworks, semantics, SCC-recursiveness, directionality

\section{Introduction}

This work lies in the general setting of abstract argumentation frameworks, as proposed by Dung [1] and deals with the characterization of argumentation semantics with respect to the local computation of extensions.

Non-interference and directionality were proposed as desirable properties of argumentation semantics, conveying the idea that for some sets of arguments the selection of arguments for an extension should not depend on the rest of the framework $[2,3]$.

SCC-recursiveness was introduced in [4] as a powerful schema for characterizing argumentation semantics with respect to the decomposition of the argumentation framework into strongly connected components (SCC's). The approach relies on the idea that the arguments selected from an SCC may only depend on arguments selected from ancestor SCC's.

* AT2012, 15-16 October 2012, Dubrovnik, Croatia. Copyright held by the author(s). 
In this paper we introduce strongly connected sets (SCS's) as a generalization of SCC's and we provide a stronger characterization of SCC-recursiveness using these sets. Furthermore, we propose SCC-directionality as a natural refinement of directionality.

We also introduce general directionality as a very broad formal approach for describing the behavior of argumentation semantics with respect to local computation of extensions and the information that needs to be available from the rest of the framework.

Section 2 introduces the argumentation concepts we are going to use, with references to the literature. We generalize SCC-recursiveness and introduce SCCdirectionality, together with its properties, in Section 3. The general directionality is presented in Section 4, with an example that outlines the added value of our approach. The paper ends with conclusions and ideas for future research in Section 5.

\section{Background}

In this section we aim to provide a minimal argumentation background, covering three aspects that are relevant to our work: argumentation semantics, SCCrecursiveness and properties of semantics. We start with a formal definition of argumentation frameworks and the related terminology.

Definition 1. An argumentation framework is a pair $F=(\mathcal{A}, \mathcal{R})$, where $\mathcal{A}$ is a set of arguments and $\mathcal{R} \subseteq \mathcal{A} \times \mathcal{A}$ is a binary attack relation on $\mathcal{A}$. Whenever $(a, b) \in \mathcal{R}$ we say that argument a attacks argument $b$ and we write this as $a \rightarrow b$. We say that a set of arguments $S \subseteq \mathcal{A}$ defends an argument a iff $S$ attacks all arguments $b$ that attack $a$. We extend the notion of attack to also refer to sets of arguments, as follows:

$$
\begin{aligned}
& a \rightarrow S \Leftrightarrow \exists b(b \in S \wedge a \rightarrow b) \\
& S \rightarrow a \Leftrightarrow \exists b(b \in S \wedge b \rightarrow a) \\
& S \rightarrow T \Leftrightarrow \exists a b(a \in S \wedge b \in T \wedge a \rightarrow b)
\end{aligned}
$$

For a given argumentation framework $F=(\mathcal{A}, \mathcal{R})$ and a set of arguments $S \subseteq \mathcal{A}$, the restriction of $F$ to $S$ is the argumentation framework

$$
F \downarrow_{S} \triangleq(S, \mathcal{R} \cap(S \times S))
$$

For example, the argumentation framework from Fig. 1 is given by $\mathcal{A}=$ $\{a, b, c, d\}$ and $\mathcal{R}=\{(a, b),(b, c),(c, d),(d, c)\}$. The restriction of $F$ to the set $\{a, b, c\}$ is $F \downarrow_{\{a, b, c\}}=(\{a, b, c\},\{(a, b),(b, c)\})$.

\subsection{Argumentation semantics}

In the argumentation literature, semantics refer to approaches (algorithmic, constraint-based or otherwise) for choosing sets of arguments (extensions) that are acceptable. We first introduce the argumentation semantics defined in [1], sometimes referred to as the traditional, or classical, semantics. 
Definition 2. Let $F=(\mathcal{A}, \mathcal{R})$ be an argumentation framework and let $E \subseteq \mathcal{A}$ be a set of arguments.

- $E$ is conflict-free iff there is no attack between arguments from $E$. The set of all conflict-free sets of $F$ is denoted by $\mathcal{E}_{\mathcal{C F}}(F)$.

- $E$ is admissible iff $E$ is conflict-free and $E$ defends all the arguments it contains. The set of all admissible sets of $F$ is denoted by $\mathcal{E}_{\mathcal{A S}}(F)$.

- $E$ is a complete extension of $F$ iff $E$ is admissible and $E$ contains all the arguments it defends. The set of all complete extensions of $F$ is denoted by $\mathcal{E}_{\mathcal{C O}}(F)$

- $E$ is a stable extension of $F$ iff $E$ is conflict-free and $E$ attacks all arguments that are not in $E$. The set of all stable extensions of $F$ is denoted by $\mathcal{E}_{\mathcal{S T}}(F)$.

- $E$ is a preferred extension of $F$ iff $E$ is a maximal (with respect to set inclusion) admissible set of $F$. The set of all preferred extensions of $F$ is denoted by $\mathcal{E}_{\mathcal{P R}}(F)$.

- $E$ is the grounded extension of $F$ iff $E$ is the (unique) minimal complete extension (with respect to set inclusion). The (singleton) set of grounded extensions is denoted by $\mathcal{E}_{\mathcal{G R}}(F)$.

The name of the sets (or extensions) presented in Definition 2 corresponds to the name of the argumentation semantics. Furthermore, whenever the extensions of a particular semantics are denoted by $\mathcal{E}_{\mathcal{S e m}}(F)$, it means that $\mathcal{S} e m$ is used as an abbreviation of the corresponding semantics. For example $\mathcal{C O}$ stands for the complete semantics.

For the framework from Fig. 1, the semantics of Definition 2 give the following extensions:

$$
\begin{aligned}
& \mathcal{E}_{\mathcal{C F}}(F)=\{\varnothing,\{a\},\{a, c\},\{a, d\},\{b\},\{b, d\},\{c\},\{d\}\} \\
& \mathcal{E}_{\mathcal{A S}}(F)=\{\varnothing,\{a\},\{a, c\},\{a, d\},\{d\}\} \\
& \mathcal{E}_{\mathcal{C O}}(F)=\{\{a\},\{a, c\},\{a, d\}\} \\
& \mathcal{E}_{\mathcal{S T}}(F)=\{\{a, c\},\{a, d\}\} \\
& \mathcal{E}_{\mathcal{P R}}(F)=\{\{a, c\},\{a, d\}\} \\
& \mathcal{E}_{\mathcal{G R}}(F)=\{\{a\}\}
\end{aligned}
$$

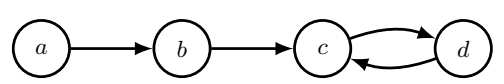

Fig. 1. Example argumentation framework.

Several other semantics have been proposed in the literature, such as ideal [5], semi-stable [6], eager [7]. Due to space constraints, we will not cover them here. 


\subsection{SCC-recursiveness}

The general idea behind SCC-recursiveness is to compute semantics taking advantage of the decomposition of the argumentation framework along its strongly connected components (SCC's). We will only provide here the minimal definitions required for introducing the concept. For more details and the rationale behind the idea, the reader may consult [4]. We start with a formal definition for the strongly connected components.

Definition 3. Let $F=(\mathcal{A}, \mathcal{R})$ be an argumentation framework. We define the path equivalence relation $P E_{F} \subseteq \mathcal{A} \times \mathcal{A}$ as follows:

- $(a, a) \in P E_{F}$ for all arguments $a \in \mathcal{A}$

- for any two arguments $a$ and $b,(a, b) \in P E_{F}$ iff there is a path in $\mathcal{R}$ from $a$ to $b$ and $a$ path from $b$ to $a$

$P E_{F}$ so defined is an equivalence relation and its equivalence classes are called strongly connected components ( $S C C^{\prime}$ 's). We will use $S C C S_{F}$ to refer to the set of strongly connected components of $F$. We will denote the strongly connected component that contains an argument a with $\operatorname{SCC}_{F}(a)$.

Given an argumentation framework $F$ and an extension $E$, we can partition the elements of any strongly connected component $S$ into three different classes, with respect to how the extension $E$ interacts with them from outside $S$. These classes are introduced in Definition 4.

Definition 4. Let $F=(\mathcal{A}, \mathcal{R})$ be an argumentation framework, $E \subseteq \mathcal{A}$ a set of arguments and $S \in S C C S_{F}$ a strongly connected component of $F$. The elements of $S$ can be partitioned into three sets with respect to $E$ :

1. $D_{F}(S, E)=\{a \in S \mid(E \backslash S) \rightarrow a\}$ - the set of arguments attacked by $E$ from outside $S$

2. $U_{F}(S, E)=\{a \in S \mid(E \backslash S) \not \rightarrow a \wedge \forall b(b \in \mathcal{A} \backslash S \wedge b \rightarrow a \Rightarrow(E \backslash S) \rightarrow b)\}$

- the set of arguments not attacked by $E$ from outside $S$ and defended by $E$ against all attackers that are not in $S$.

3. $P_{F}(S, E)=S \backslash\left(D_{F}(S, E) \cup U_{F}(S, E)\right)$ - arguments that are neither attacked by $E$ from outside of $S$, nor defended by $E$ against attacks coming from outside $S$.

With the use of these sets, SCC-recursive semantics can be defined. We will use $U P_{F}(S, E)$ as an abbreviation for $U_{F}(S, E) \cup P_{F}(S, E)$, which is the same as $S \backslash D_{F}(S, E)$. Note that in the formulas from Definition 4 we have used $\Rightarrow$ for logical implication so that there is no confusion with the attack relation, denoted by $\rightarrow$.

Definition 5. An argumentation semantics $\mathcal{S} e m$ is said to be $\boldsymbol{S C C}$-recursive iff, for any argumentation framework $F=(\mathcal{A}, \mathcal{R}), \mathcal{E}_{\mathcal{S e m}}(F)=\mathcal{G} \mathcal{F}(F, \mathcal{A})$, where the generic recursive function $\mathcal{G} \mathcal{F}$ is defined as follows: for any argumentation framework $F=(\mathcal{A}, \mathcal{R})$ and any two sets of arguments $E, C \subseteq \mathcal{A}$ it holds that $E \in \mathcal{G} \mathcal{F}(F, C)$ iff 
- in case $\left|S C C S_{F}\right|=1, E \in \mathcal{B F}_{\mathcal{S e m}}(F, C)$

- otherwise, for all strongly connected components $S \in S C C S_{F}$, we have $(E \cap S) \in \mathcal{G F}\left(F \downarrow_{U P_{F}(S, E)}, C \cap U_{F}(S, E)\right)$

where $\mathcal{B F}_{\mathcal{S}_{e m}}$ is a base function that depends on $\mathcal{S}$ em.

The idea conveyed in Definition 5 is that the arguments chosen from a strongly connected component $S$ as elements of an extension $E$ are selected based on what has already been selected from other SCC's, more precisely by taking into account which arguments are defended $\left(U_{F}(S, E)\right)$ or at least not defeated $\left(U P_{F}(S, E)\right)$ by the arguments selected for $E$ from components that attack $S$.

It is shown in [4] that all the classical semantics are SCC-recursive so this approach proves to be an alternative to their original definitions.

We can use the definition of SCC-recursiveness in two ways for computing extensions. We can consider all possible sets of arguments and check whether the property specified in the definition holds or we can compute extensions iteratively, starting with unattacked SCC's and considering components one by one.

Definition 6. The $\mathcal{C} \mathcal{F} 2$ semantics [4] is the $S C C$-recursive semantics given by the following base function:

$$
\mathcal{B F}_{C F 2}(F, C)=\mathcal{E}_{\mathcal{M C F}}(F)
$$

where $\mathcal{E}_{\mathcal{M C F}}(F)$ stands for the set of maximal (with respect to set inclusion) conflict-free sets, also known as naive extensions.

For the framework from Fig. 1 we have $\mathcal{E}_{\mathcal{M C F}}(F)=\{\{a, b\},\{a, c\},\{b, d\}\}$. Let us also discuss the extensions of $\mathcal{C F} 2$. The strongly connected components of $F$ are $S_{1}=\{a\}, S_{2}=\{b\}$ and $S_{3}=\{c, d\}$. The extensions are $\mathcal{E}_{\mathcal{C F} 2}(F)=$ $\{\{a, c\},\{a, d\}\}$. Let us see that $E=\{a, c\}$ is indeed a $\mathcal{C F} 2$ extension. We will use $\mathcal{C F} 2(F, C)$ instead of $\mathcal{G} \mathcal{F}(F, C)$ for the recursive function. So we want to show that $E \in \mathcal{C} \mathcal{F} 2(F, \mathcal{A})$. We have:

$$
\begin{aligned}
E \cap S_{1}=\{a\} & \in \mathcal{C F} 2\left(F \downarrow_{U P_{F}\left(S_{1}, E\right)}, \mathcal{A} \cap U_{F}\left(S_{1}, E\right)\right) \\
& =\mathcal{C F} 2\left(F \downarrow_{S_{1}}, S_{1}\right)=\{\{a\}\} \\
E \cap S_{2}=\varnothing & \in \mathcal{C F} 2\left(F \downarrow_{U P_{F}\left(S_{2}, E\right)}, \mathcal{A} \cap U_{F}\left(S_{2}, E\right)\right) \\
& =\mathcal{C F} 2\left(F \downarrow_{\varnothing}, \varnothing\right)=\{\varnothing\} \\
E \cap S_{3}=\{c\} & \in \mathcal{C F} 2\left(F \downarrow_{U P_{F}\left(S_{3}, E\right)}, \mathcal{A} \cap U_{F}\left(S_{3}, E\right)\right) \\
& =\mathcal{C F} 2\left(F \downarrow_{S_{3}}, S_{3}\right)=\{\{c\},\{d\}\}
\end{aligned}
$$

So the requirement from Definition 5 is satisfied. 


\subsection{Properties of Argumentation Semantics}

A principle-based evaluation of argumentation semantics was proposed in [2] and extended in [3]. We only provide formal definitions here for the properties that are relevant for this work.

Definition 7. An argumentation semantics $\mathcal{S}$ em is universally defined iff, for any argumentation framework $F, \mathcal{E}_{\mathcal{S e m}}(F) \neq \varnothing$.

In words, universally defined argumentation semantics provide at least one extension for any argumentation framework. Of the semantics we have presented here, only $\mathcal{S} \mathcal{T}$ is not universally defined [2]

Definition 8. Let $F=(\mathcal{A}, \mathcal{R})$ be an argumentation framework. A set of arguments $S \subseteq \mathcal{A}$ is isolated iff there are no attacks between $S$ and the rest of the framework:

$$
\mathcal{R} \cap((S \times(\mathcal{A} \backslash S)) \cup((\mathcal{A} \backslash S) \times S))=\varnothing
$$

The set of all isolated sets of $F$ is denoted by $\mathcal{I S}(F)$.

An argumentation semantics $\mathcal{S} e m$ is said to satisfy the non-interference principle iff, for any argumentation framework $F=(\mathcal{A}, \mathcal{R})$ and every isolated set $S \in \mathcal{I S}(F)$ it holds that:

$$
\mathcal{A E}_{\mathcal{S e m}}(F, S)=\mathcal{E}_{\mathcal{S e m}}\left(F \downarrow_{S}\right)
$$

where $\mathcal{A E}_{\mathcal{S e m}}(F, S) \triangleq\left\{E \cap S \mid E \in \mathcal{E}_{\mathcal{S e m}}(F)\right\}$

The intuition behind non-interference is that the elements chosen for an extension $E$ from an isolated set $S$ can be computed locally in the restricted framework $F \downarrow_{S}$ by using the same argumentation semantics. Furthermore, for any such locally computed extension, it should be possible to select additional arguments from the rest of the framework so as to get an extension of $F$. Again, of the semantics we have formally introduced, only $\mathcal{S T}$ fails to satisfy noninterference.

Definition 9. Let $F=(\mathcal{A}, \mathcal{R})$ be an argumentation framework. A set of arguments $S$ is unattacked iff $S$ is not attacked by any argument that is not in $S$ :

$$
\mathcal{A} \backslash S \not \rightarrow S
$$

The set of all unattacked sets of $F$ is denoted by $\mathcal{U S}(F)$.

An argumentation semantics $\mathcal{S}$ em satisfies the directionality principle iff, for any argumentation framework $F=(\mathcal{A}, \mathcal{R})$ and any unattacked set $S \in$ $\mathcal{U S}(F)$, it holds that:

$$
\mathcal{A E}_{\mathcal{S e m}}(F, S)=\mathcal{E}_{\mathcal{S e m}_{e m}}\left(F \downarrow_{S}\right)
$$

Note that directionality and non-interference impose the same constraint, the only difference is the sets for which the relation must hold. Furthermore, directionality implies non-interference [3]. The stable semantics does not satisfy 
directionality [2], nor does the naive semantics [8]. The other semantics discussed in this paper do satisfy this property.

If we think of SCC-recursiveness as a property as well, we have already mentioned that all classical semantics satisfy it. Furthermore, $\mathcal{C} \mathcal{F} 2$ satisfies it by definition. On the other hand, it is shown in [2] that all SCC-recursive semantics that are universally defined satisfy directionality. Since $\mathcal{M C F}$ does not satisfy directionality, it follows that it is not SCC-recursive either. On the other hand, ideal semantics satisfies directionality [2], but is not SCC-recursive [9], so the two properties are indeed distinct. Table 1 summarizes the results we have mentioned in this section.

\begin{tabular}{|c|c|c|c|c|c|c|c|c|}
\hline Semantics & $\mathcal{C F}$ & $\mathcal{A S}$ & $\mathcal{C O}$ & $\mathcal{S T}$ & $\mathcal{P R}$ & $\mathcal{G R}$ & $\mathcal{M C F}$ & $\mathcal{C F} 2$ \\
\hline Universally defined & Yes & Yes & Yes & No & Yes & Yes & Yes & Yes \\
\hline Non-interference & Yes & Yes & Yes & No & Yes & Yes & Yes & Yes \\
\hline Directionality & Yes & Yes & Yes & No & Yes & Yes & No & Yes \\
\hline SCC-recursiveness & Yes & Yes & Yes & Yes & Yes & Yes & No & Yes \\
\hline
\end{tabular}

Table 1. Satisfaction of the evaluation principles.

In our work, we were inspired by the similarities that exist between noninterference, directionality and SCC-recursiveness with respect to what they describe as reasonable for the intersection of an extension with a set of arguments.

\section{SCC-directionality}

We have seen that non-interference and directionality require that the intersection of an extension with an isolated (or unattacked) set $S$ can be computed as an extension of the restricted framework $F \downarrow_{S}$. In this section we wish to define a new type of sets, one that includes unattacked and isolated sets but also generalizes strongly connected components, then formulate a principle about the intersection of extensions with such sets.

Definition 10. Let $F=(\mathcal{A}, \mathcal{R})$ be an argumentation framework and let $S \subseteq \mathcal{A}$ be a set of arguments. $S$ is a strongly connected set iff, for all arguments $a \in \mathcal{A}$, if a indirectly attacks $S$ and $S$ indirectly attacks $a$, then a is in $S$. We will use $\mathcal{S C S}(F)$ to refer to all strongly connected sets of $F$.

$$
\mathcal{S C S}(F)=\left\{S \subseteq \mathcal{A} \mid \forall a\left(a \rightarrow^{*} S \wedge S \rightarrow^{*} a \Rightarrow a \in S\right)\right\}
$$

where $\rightarrow^{*}$ stands for a path of attacks (one or more).

Proposition 1. Let $F=(\mathcal{A}, \mathcal{R})$ be an argumentation framework. Every strongly connected set $S \in \mathcal{S C S}(F)$ can be written as the union of zero or more strongly connected components of $F$. 
Proof. Given an argument $a \in S$, for all arguments $b \in S C S_{F}(a)$ we have that $a \rightarrow^{*} b$ and $b \rightarrow^{*} a$, which leads to $b \in S$ and, thus, $S C C_{F}(a) \subseteq S$. It follows that $S=\bigcup_{a \in S} S C C_{F}(a)$.

Next, we introduce several notations and concepts that will help provide another characterization for strongly connected sets, one that gives a better intuition about the actual property of SCC's that is preserved for strongly connected sets.

Definition 11. Let $F=(\mathcal{A}, \mathcal{R})$ be an argumentation framework and let $S \subseteq \mathcal{A}$ be a set of arguments. We introduce the following notations:

$$
\begin{aligned}
& \mathcal{L A}_{1}(F, S)=\left\{T \in S C C S_{F} \mid T \nsubseteq S \wedge S \rightarrow T\right\} \\
& \mathcal{L B}_{1}(F, S)=\left\{T \in S C C S_{F} \mid T \nsubseteq S \wedge T \rightarrow S\right\} \\
& \mathcal{L} \mathcal{A}_{n+1}(F, S)=\mathcal{L A}_{1}\left(F, S \cup \bigcup_{i=1}^{n} \mathcal{L A}_{i}(F, S)\right) \\
& \mathcal{L B}_{n+1}(F, S)=\mathcal{L B}_{1}\left(F, S \cup \bigcup_{i=1}^{n} \mathcal{L B}_{i}(F, S)\right) \\
& \mathcal{L} \mathcal{A}^{*}(F, S)=\bigcup_{n \geq 1} \mathcal{L} \mathcal{A}_{n}(F, S) \\
& \mathcal{L} \mathcal{B}^{*}(F, S)=\bigcup_{n \geq 1} \mathcal{L B}_{n}(F, S)
\end{aligned}
$$

These notations correspond to the following concepts:

- $\mathcal{L} \mathcal{A}_{n}$ - level $n$ look-ahead of $S$ in $F$

- $\mathcal{L B}_{n}$ - level $n$ look-back of $S$ in $F$

- $\mathcal{L A}^{*}$ - total look-ahead of $S$ in $F$

- $\mathcal{L B}^{*}$ - total look-back of $S$ in $F$

Note that for arbitrary sets it is possible that $S \cap \mathcal{L} \mathcal{A}_{1}(F, S) \neq \varnothing$ and $S \cap$ $\mathcal{L} \mathcal{A}^{*}(F, S) \neq \varnothing$. On the other hand, whenever $S$ is the union of zero or more strongly connected components, $S \cap \mathcal{L A}_{1}(F, S)=\varnothing$ follows from the definition, since $T \nsubseteq S$ and $T$ is a strongly connected component. Similar considerations apply to the look-back.

Proposition 2. Let $F=(\mathcal{A}, \mathcal{R})$ be an argumentation framework and let $S \subseteq \mathcal{A}$ be a set of arguments. The following relations hold:

(a) $S \in \mathcal{I S}(F) \Leftrightarrow \mathcal{L A}^{*}(F, S)=\mathcal{L B}^{*}(F, S)=\varnothing$

(b) $S \in \mathcal{U S}(F) \Leftrightarrow \mathcal{L B}^{*}(F, S)=\varnothing$

(c) $S \in \mathcal{S C S}(F) \Leftrightarrow \mathcal{L A}^{*}(F, S) \cap \mathcal{L B}^{*}(F, S)=\varnothing$

The alternative characterizations from Proposition 2 follow from the corresponding definitions. The advantage of using look-back and look-ahead consists in clustering the attackers of $S$ and the arguments attacked by $S$ into strongly connected components and distinguishing between the ones that are closer to $S$ and those that are farther away.

Note that only one-way attacks are possible between a strongly connected set and a strongly connected component. On the other hand, this is not necessarily the case for two SCS's. Indeed, suppose we have four SCC's: $S_{1}, S_{2}, S_{3}$ and $S_{4}$ and the attacks between them are $S_{1} \rightarrow S_{2}$ and $S_{3} \rightarrow S_{4}$. Then the strongly connected sets $S_{1} \cup S_{4}$ and $S_{2} \cup S_{3}$ attack each other. 
Corollary 1. Let $F=(\mathcal{A}, \mathcal{R})$ be an argumentation framework. All isolated sets of $F$ are also unattacked and all unattacked sets of $F$ are also strongly connected sets in $F$

$$
\mathcal{I S}(F) \subseteq \mathcal{U S}(F) \subseteq \mathcal{S C S}(F)
$$

Furthermore, let us see that, for any set $S$, the set $S \cup \mathcal{L B}^{*}(F, S)$ is an unattacked set (it can be shown that it is the minimal unattacked set that contains $S$ ). Similarly, $S \cup \mathcal{L A}^{*}(F, S) \cup \mathcal{L B}^{*}(F, S)$ is the minimal isolated set that contains $S$.

We are now ready to prove one of the main results of this paper, the generalization of SCC-recursiveness.

Theorem 1. Let $\mathcal{G F}$ be the generic function of an $S C C$-recursive argumentation semantics. Then, for any argumentation framework $F=(\mathcal{A}, \mathcal{R})$ and for any partition of $\mathcal{A}$ into disjoint strongly connected sets $\mathcal{P}$ art $=\left\{S_{1}, S_{2}, \ldots, S_{n}\right\}$, the following holds:

$$
E \in \mathcal{G F}(F, C) \Leftrightarrow \forall S\left(S \in \mathcal{P} a r t \Rightarrow(E \cap S) \in \mathcal{G} \mathcal{F}\left(F \downarrow_{U P_{F}(S, E)}, C \cap U_{F}(S, E)\right)\right)
$$

Proof. We know that the result holds if the partition consists of all the SCC's of $F$. What we need to show is that it holds for other partitions as well. We proceed by induction on the size of $F$. For frameworks with a single argument there will be a single SCC and a single partition into strongly connected sets, so the result holds trivially.

For the induction step, we assume that the claim is true for all frameworks that have fewer arguments than $F$ and we prove it for $F$. We start with the converse. Let us see that if $\mathcal{P}$ art $=\{\mathcal{A}\}$ the claim trivially holds. In what follows we focus on partitions containing at least two distinct strongly connected sets. We must prove that if $E \cap S \in \mathcal{G} \mathcal{F}\left(F \downarrow_{U P_{F}(S, E)}, C \cap U_{F}(S, E)\right)$ for all $S \in \mathcal{P}$ art, then $E \in \mathcal{G F}(F, C)$.

Let $S \in \mathcal{P}$ art be an arbitrary SCS. Then $E \cap S \in \mathcal{G F}\left(F \downarrow_{U P_{F}(S, E)}, C \cap\right.$ $\left.U_{F}(S, E)\right)$. We know from Proposition 1 that $S$ is the union of one or more SCC's of $F$. For any such component $T$, we have that $T \cap U P_{F}(S, E)$ is a strongly connected set in $F \downarrow_{U P_{F}(S, E)}$, so we can apply the induction hypothesis for $F^{\prime}=$ $F \downarrow_{U P_{F}(S, E)}$ and the extension $E \cap S$. We get $(E \cap S) \cap\left(T \cap U P_{F}(S, E)\right) \in$ $\mathcal{G F}\left(F^{\prime} \downarrow_{U P_{F^{\prime}}\left(T \cap U P_{F}(S, E), E \cap S\right)}, C \cap U_{F}(S, E) \cap U_{F^{\prime}}\left(T \cap U P_{F}(S, E), E \cap S\right)\right)$.

Since $E \cap S \subseteq U P_{F}(S, E)$ and $T \subseteq S$, we can write $(E \cap S) \cap\left(T \cap U P_{F}(S, E)\right)=$ $E \cap T$. Furthermore, let us see that $U P_{F^{\prime}}\left(T \cap U P_{F}(S, E), E \cap S\right)=U P_{F}(T, E)$. Indeed, for any argument $a \in \mathcal{A}$, we have $a \in U P_{F^{\prime}}\left(T \cap U P_{F}(S, E), E \cap S\right) \Leftrightarrow$ $a \in T \wedge a \in U P_{F}(S, E) \wedge\left((E \cap S) \backslash\left(T \cap U P_{F}(S, E)\right)\right) \not \rightarrow a \Leftrightarrow a \in T \wedge(E \backslash S) \not \rightarrow$ $a \wedge((E \cap S) \backslash T) \not \rightarrow a \Leftrightarrow a \in T \wedge(E \backslash T) \not \rightarrow a \Leftrightarrow a \in U P_{F}(T, E)$.

Similarly, we show that $U_{F}(S, E) \cap U_{F^{\prime}}\left(T \cap U P_{F}(S, E), E \cap S\right)=U_{F}(T, E)$. Here, we actually need to prove the double inclusion. We start with $\subseteq$. Let $a$ be an argument from $U_{F}(S, E) \cap U_{F^{\prime}}\left(T \cap U P_{F}(S, E), E \cap S\right)$ and let $b$ be an attacker of $a$, with $b \in \mathcal{A} \backslash T$. If $b \notin S$, then $(E \backslash S) \rightarrow b$, because $a \in U_{F}(S, E)$. If $b \in S$, we have either $b \in D_{F}(S, E)$, in which case $(E \backslash S) \rightarrow b$, or $b \in U P_{F}(S, E)$ and 
then $(E \cap S) \backslash\left(T \cap U P_{F}(S, E)\right) \rightarrow b$ because $a \in U_{F^{\prime}}\left(T \cap U P_{F}(S, E), E \cap S\right)$. In all three cases we can infer $(E \backslash T) \rightarrow b$. Thus, we conclude that $a \in U_{F}(T, E)$.

For the other inclusion, let us consider $a \in U_{F}(T, E)$ and $b$ an attacker of $a$. If $b \in U P_{F}(S, E) \backslash T$. Since $a \in U_{F}(T, E)$, we have $(E \backslash T) \rightarrow b$. But $b \notin D_{F}(S, E)$, so $(E \backslash S) \not \rightarrow b$, which leads to $((E \cap S) \backslash T) \rightarrow b$, so $a \in U_{F^{\prime}}\left(T \cap U P_{F}(S, E), E \cap S\right)$. If $b \in \mathcal{A} \backslash S$ we still have $(E \backslash T) \rightarrow b$, but what we need for $a \in U_{F}(S, E)$ is $(E \backslash S) \rightarrow b$. Suppose that this is not the case, i.e. there exists $c \in(E \cap S) \backslash T$ such that $c \rightarrow b$. Since both $c$ and $a$ are in $S$, we have $S \rightarrow^{*} b$ and $b \rightarrow^{*} S$ so we must have $b \in S$ because $S$ is strongly connected. However, this contradicts the assumption that $b \in \mathcal{A} \backslash S$.

Putting it all together, we got $E \cap T \in \mathcal{G} \mathcal{F}\left(F \downarrow_{U P_{F}(T, E)}, C \cap U_{F}(T, E)\right)$. And this holds for every SCS $S \in \mathcal{P}$ art and every SCC $T \subseteq S$. Since Part is a partition, this covers all possible SCC's of $F$ so, based on the SCC-recursiveness of $\mathcal{S} e m$, we can conclude that $E \in \mathcal{G} \mathcal{F}(F, C)$, as desired.

We now discuss the direct implication. So we have $E \in \mathcal{G} \mathcal{F}(F, C)$. Just as before, we consider $S \in \mathcal{P}$ art and $T \in S C C S_{F}$ such that $T \subseteq S$. From SCCrecursiveness we have $E \cap T \in \mathcal{G} \mathcal{F}\left(F \downarrow_{U P_{F}(T, E)}, C \cap U_{F}(T, E)\right)$. We now use the same equalities as above in order to obtain $(E \cap S) \cap\left(T \cap U P_{F}(S, E)\right) \in$ $\mathcal{G} \mathcal{F}\left(F \downarrow_{U_{F^{\prime}}\left(T \cap U P_{F}(S, E), E \cap S\right)}, C \cap U_{F}(S, E) \cap U_{F^{\prime}}\left(T \cap U P_{F}(S, E), E \cap S\right)\right)$. Since $F^{\prime}$ has fewer arguments than $F$, we use the induction hypothesis and we get $E \cap S \in \mathcal{G} \mathcal{F}\left(F \downarrow_{U P_{F}(S, E)}, C \cap U_{F}(S, E)\right)$. This completes our proof.

The very strong result covered by Theorem 1 reveals that we don't need to split a framework along its SCC's, it is enough to choose any partition into strongly connected sets in order to get the same property as that required by SCC-recursiveness. On a more practical note, this also means that whenever we put two frameworks together and only add attacks from one of them towards the other, we can compute the extensions of the union framework using the SCC-recursive approach. The theorem also has two immediate corollaries.

Corollary 2. Let $F=(\mathcal{A}, \mathcal{R})$ be an argumentation framework and let $\mathcal{G} \mathcal{F}$ be the generic function of an SCC-recursive argumentation semantics. For every $E, C \subseteq \mathcal{A}$, the following relation holds:

$$
E \in \mathcal{G} \mathcal{F}(F, C) \Leftrightarrow \forall S\left(S \in \mathcal{S C S}(F) \Rightarrow(E \cap S) \in \mathcal{G F}\left(F \downarrow_{U P_{F}(S, E)}, C \cap U_{F}(S, E)\right)\right)
$$

Corollary 3. Let $F=(\mathcal{A}, \mathcal{R})$ be an argumentation framework and let $\mathcal{S}$ em be an $S C C$-recursive argumentation semantics whose generic function does not use its second argument. Then, for every set of arguments E, we have:

$$
\left.E \in \mathcal{E}_{\mathcal{S e m}_{e m}}(F) \Leftrightarrow \forall S\left(S \in \mathcal{S C S}(F) \Rightarrow(E \cap S) \in \mathcal{E}_{\mathcal{S e m}_{e}\left(F \downarrow_{U P_{F}}(S, E)\right.}\right)\right)
$$

Let us see that the result in Corollary 3 is somewhat similar to the condition that characterizes both non-interference and directionality, in the sense that the same semantics is applied for a restricted framework. Based on this similarity, we formalize the idea of SCC-directionality. 
Note however that, while both non-interference and directionality state that the local computation of an extension (its intersection with an isolated or unattacked set) should be computable independently of the rest of the framework, the core intuition behind SCC-recursiveness is quite different. What we need to say for strongly connected sets is that the local computation is reasonably influenced by the computation performed for some other set of arguments.

Definition 12. Let $F=(\mathcal{A}, \mathcal{R})$ be an argumentation framework. An argumentation semantics $\mathcal{S} e m$ satisfies the $\boldsymbol{S C C}$-directionality principle iff, for any strongly connected set $S \in \mathcal{S C S}(F)$ and any $T \in \mathcal{A E}_{\mathcal{S e m}}\left(F, \mathcal{L B}^{*}(F, S)\right)$, we have

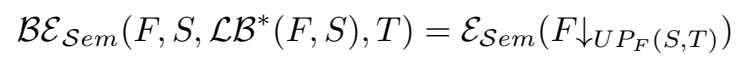

where, for any sets of arguments $S, D, T$, we define

$$
\mathcal{B E}_{\mathcal{S}_{\text {Sem }}}(F, S, D, T) \triangleq\left\{E \cap S \mid E \in \mathcal{E}_{\mathcal{S e m}}(F) \wedge E \cap D=T\right\}
$$

The meaning of SCC-directionality is that, given a strongly connected set $S$, the arguments we can choose from $S$ for an extension $E$ are determined by what we have chosen in $E$ from the total look-back of $S$ and, furthermore, for any meaningful choice that we can make in $\mathcal{L B}^{*}(F, S)$, the choices from $S$ can be computed using the same semantics, but applied to a restricted framework that accounts for the selection $T$.

Note that the significant novelty of SCC-directionality lies more in the use of strongly connected sets and the actual formalization using $\mathcal{B E}$ than in the total look-back, which is in fact equivalent to the union of all ancestor SCC's that are mentioned in [4].

Proposition 3. Every universally defined SCC-recursive semantics Sem whose generic function does not use its second argument satisfies SCC-directionality.

Proof. Let $F=(\mathcal{A}, \mathcal{R})$ be an argumentation framework and let $S \in \mathcal{S C S}(F)$ be an arbitrary strongly connected set. Also, let $T \in \mathcal{A E}_{\mathcal{S e m}}\left(F, \mathcal{L B}^{*}(F, S)\right)$. Given $U \in \mathcal{B E}_{\mathcal{S e m}}\left(F, S, \mathcal{L B}^{*}(F, S), T\right)$, we have that there exists $E \in \mathcal{E}_{\mathcal{S e m}}(F)$ such that $E \cap \mathcal{L B}^{*}(F, S)=T$ and $E \cap S=U$. Furthermore, from Corollary 3 we have that $E \cap S \in \mathcal{E}_{\mathcal{S e m}}\left(F \downarrow_{U P_{F}(S, E)}\right)$. However, let us see that, in fact, $U P_{F}(S, E)=U P_{F}\left(S, E \cap \mathcal{L B}^{*}(F, S)\right)$, which leads to $U P_{F}(S, E)=U P_{F}(S, T)$. Thus, we have proved that $\mathcal{B E}_{\mathcal{S e m}}\left(F, S, \mathcal{L B}^{*}(F, S), T\right) \subseteq \mathcal{E}_{\mathcal{S e m}}\left(F \downarrow_{U P_{F}(S, T)}\right)$.

For the other inclusion, let us consider $U \in \mathcal{E}_{\mathcal{S e m}}\left(F \downarrow_{U P_{F}(S, T)}\right)$. We denote $S_{1}=S \cup \mathcal{L B}^{*}(F, S)$ and $S_{2}=\mathcal{A} \backslash S_{1}$ and take the partition Part $=\left\{S_{1}, S_{2}\right\}$. Both elements are strongly connected sets and, since $\mathcal{S} e m$ is universally defined, we have $\mathcal{E}_{\mathcal{S e m}}\left(F \downarrow_{U P_{F}\left(S_{2}, T \cup U\right)}\right) \neq \varnothing$ so we can choose an extension $V$ from this set. but then $T \cup U \cup V$ satisfies the conditions of Theorem 1, which leads to $T \cup U \cup V \in \mathcal{E}_{\mathcal{S e m}}(F)$.

The previous result covers the $\mathcal{C F}$ and $\mathcal{C F} 2$ semantics. For the other semantics, let us consider the argumentation framework from Fig. 2 and see that SCC-directionality is violated for $\mathcal{A S}$. We consider $S=\{c, d\}$. Then $\mathcal{L B}^{*}(F, S)=$ 
$\{a, b, e\}$. Since $\{a, c\}$ is admissible, we have that $\{a\} \in \mathcal{A E}_{\mathcal{A S}}\left(F, \mathcal{L B}^{*}(F, S)\right)$, so we can choose $T=\{a\}$. But then $\mathcal{E}_{\mathcal{A S}}\left(F \downarrow_{U P_{F}(S, T)}\right)=\{\varnothing,\{c\},\{d\}\}$. However, there is no admissible set that contains $d$. The proof for the other semantics based on admissibility $(\mathcal{C O}, \mathcal{S T}, \mathcal{G} \mathcal{R}$ and $\mathcal{P} \mathcal{R})$ is similar.

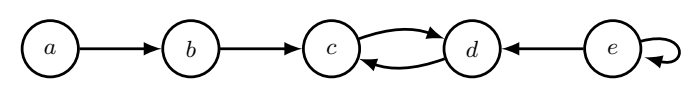

Fig. 2. Argumentation framework showing that several semantics do not satisfy SCCdirectionality.

Furthermore, let us see that, for universally defined semantics, the noninterference and directionality principles are just special cases of the newly introduced SCC-directionality, where the relation is required to hold only for isolated (respectively unattacked) sets. The proof relies on the fact that, for unattacked sets, $\mathcal{B E}_{\mathcal{S e m}}\left(F, S, \mathcal{L B}^{*}(F, S)\right)=\mathcal{A E}(F, S)$ and $U P_{F}(S, T)=S$.

\section{General directionality}

In this section we introduce general directionality, based on the intuition that, for certain sets of arguments $S$, the intersection of an extension $E$ with $S$ depends on some set of arguments $D$ in the sense that this intersection can be computed locally, using a generic function that works with information compiled from the intersection of $E$ with $D$. We formalize this in Def. 13.

Definition 13. An argumentation semantics $\mathcal{S} e m$ is said to satisfy general directionality with signature $\Delta(\mathcal{S}$ ets, Det, Local, Info $)$ iff, for any argumentation framework $F=(\mathcal{A}, \mathcal{R})$, any set $S \in \mathcal{S} \operatorname{Sets}(F)$ and any set $T \in$ $\mathcal{A E}_{\mathcal{S e m}}(F, \operatorname{Det}(F, S))$, the following holds:

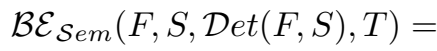

$$
\begin{aligned}
& \mathcal{G F}_{\mathcal{S e m}}\left(F \downarrow_{\mathcal{L} \operatorname{Loal}(F, S)}, \operatorname{Info}\left(F \downarrow_{\operatorname{Det}(F, S) \cup \mathcal{L} o c a l(F, S)}, S, T\right)\right)
\end{aligned}
$$

where:

$-\mathcal{G F}_{\mathcal{S} e m}$ is a function that depends on $\mathcal{S} e m$ (and on the signature of the general directionality)

- Sets $(F)$ returns the sets of arguments $S$ for which the relation holds. For example, $\mathcal{S}$ ets $\in\{\mathcal{I} \mathcal{S}, \mathcal{U S}, \mathcal{S C S}\}$

- Det $(F, S)$ returns the set of arguments that reasonably influences the intersection of extensions with $S$; it must satisfy $\operatorname{Det}(F, S) \cap S \neq \varnothing$. For example, $\mathcal{D}$ et $\in\left\{\mathcal{L B}_{n}, \mathcal{L B}^{*}, \mathcal{L A}_{n}, \mathcal{L A}^{*}, \mathcal{L B}_{1} \cup \mathcal{L} \mathcal{A}_{1}\right\}$

- $\mathcal{L}$ ocal $(F, S)$ gives the set that can be used for restricting the framework that is available to $\mathcal{G F}$; by convention, we use $\mathcal{L S}(F, S)=S$ and $\mathcal{A l l}(F, S)=\mathcal{A}$. For example $\mathcal{L}$ ocal $\in\left\{\mathcal{L S}, \mathcal{L} \mathcal{S} \cup \mathcal{L B}_{1}\right\}$ 
- Info $(F, S, T)$ encodes the knowledge of $T$ into information that is included in $\mathcal{L}$ ocal $(F, S)$; based on the way we used it in the definition, this information should be computable in the restriction of $F$ to $\mathcal{L}$ ocal $(F, S) \cup \operatorname{Det}(F, S)$. Furthermore, we define the following $\mathcal{D}(F, S, T)=D_{F}(S, T), \mathcal{U}(F, S, T)=$ $U_{F}(S, T), \mathcal{S}(F, S, T)=S$ and $\mathcal{T}(F, S, T)=T$.

Proposition 4. Any argumentation semantics $\mathcal{S} e m$ satisfies $\Delta(\mathcal{S}$ ets, $\mathcal{D}$ et, $\mathcal{A l l}$, $(\mathcal{S}, \mathcal{T}, \mathcal{D} e t))$-directionality, for any $\mathcal{S} e t s$ and $\mathcal{D}$ et

Proof. We can take $\mathcal{G} \mathcal{F}_{\mathcal{S e m}}(F,(S, T, \operatorname{Det}(F, S)))=\mathcal{B E}_{\mathcal{S e m}}(F, S, \operatorname{Det}(F, S), T)$.

Proposition 5. For universally defined semantics, SCC-directionality is equivalent to $\Delta\left(\mathcal{S C S}, \mathcal{L B}^{*}, \mathcal{L S},(\mathcal{S}, \mathcal{D})\right)$-directionality.

Proof. The result follows from the following relation $U P_{F}(S, T)=S \backslash D_{F}(S, T)=$ $S \backslash D_{\bigvee_{S \cup \mathcal{L B} \mathcal{B}^{*}(F, S)}}(S, T)$.

Furthermore, it is easy to see that we can get the equivalent characterization of non-interference and directionality in the general directionality setting by replacing $\mathcal{S C S}$ with $\mathcal{I S}$, respectively $\mathcal{U S}$ in the signature.

We also provide the following result, without proof:

Proposition 6. Any $S C C$-recursive argumentation semantics that is universally defined satisfies $\Delta\left(\mathcal{S C S}, \mathcal{L B}^{*}, \mathcal{L S},(\mathcal{D}, \mathcal{U})\right)$-directionality.

If we note that the actual computation of $D_{F}$ and $U_{F}$ only depends on the parent SCC's of $S$, we can refine the results in Proposition 5 and Proposition 6 by replacing $\mathcal{L B}^{*}$ with $\mathcal{L B}_{1}$.

If we compare SCC-recursiveness with respect to the use of the second argument (the defense information), we can see that our model for describing directionality properties clearly shows the distinction between the two classes of SCC-recursiveness, namely the defense information $\mathcal{U}$. Furthermore, the defeat information, which is somewhat hidden in the original definition of SCCrecursiveness, is present in our model.

All the results presented so far were based on the look-back information. A simple example that uses forward information can be given for conflict-free sets:

Proposition 7. $\mathcal{C F}$ satisfies $\Delta\left(\mathcal{S C S}, \mathcal{L} \mathcal{A}_{1}, \mathcal{L S},\left(\mathcal{D}^{\prime}\right)\right)$, where $\mathcal{D}^{\prime}(F, S, T)=\{a \in$ $S \mid a \rightarrow(T \backslash S)\}$.

Proof. Conflict-free sets are independent of the direction of the attacks. If all attacks are reversed, the meaning of forward and backward information interchanges, while the defeat information $\mathcal{D}$ becomes $\mathcal{D}^{\prime}$. 


\section{Conclusions and Future Work}

The first contribution of this paper is the generalization of strongly connected components as strongly connected sets and their use for a stronger version of SCC-recursiveness. We have also turned the simple SCC-recursiveness (without defense information) into a directionality-like property that refines plain directionality.

Based on the similarities and differences between non-interference, directionality and SCC-recursiveness, we have provided in this paper a unifying model that can capture these properties and at the same time provide the means for comparing such properties with respect to various aspects.

The fact that all argumentation semantics satisfy some (trivial) form of general directionality suggests the possibility of searching for "minimal" kinds of directionality that are satisfied by each semantics. For SCC-recursiveness we have seen already that ancestor information can be replaced with just parent information.

Future work will explore the relations between various kinds of general directionality. We will also give more attention to the look-ahead information, since it might be relevant for argumentation semantics such as stage or semi-stable.

\section{Acknowledgement}

This work has been funded by the Sectoral Operational Programme Human Resources Development 2007-2013 of the Romanian Ministry of Labour, Family and Social Protection through the Financial Agreement POSDRU/88/1.5/S/61178 and by project ERRIC (Empowering Romanian Research on Intelligent Information Technologies), number 264207/FP7-REGPOT-2010-1.

\section{References}

1. Dung, P.M.: On the acceptability of arguments and its fundamental role in nonmonotonic reasoning, logic programming and n-person games. Artificial Intelligence $\mathbf{7 7}(2)$ (1995) 321-357

2. Baroni, P., Giacomin, M.: On principle-based evaluation of extension-based argumentation semantics. Artificial Intelligence 171(10-15) (July 2007) 675-700

3. Baroni, P., Caminada, M., Giacomin, M.: An introduction to argumentation semantics. The Knowledge Engineering Review 26(4) (December 2011) 365-410

4. Baroni, P., Giacomin, M., Guida, G.: SCC-recursiveness: a general schema for argumentation semantics. Artificial Intelligence 168(1-2) (October 2005) 162-210

5. Dung, P.M., Mancarella, P., Toni, F.: Computing ideal sceptical argumentation. Artificial Intelligence 171(10-15) (July 2007) 642-674

6. Caminada, M.: Semi-stable semantics. In Dunne, P.E., Bench-Capon, T.J.M., eds.: Computational Models of Argument: Proceedings of COMMA 2006. Volume 144 of Frontiers in Artificial Intelligence and Applications., IOS Press (September 11-12 2006) 121-130 
7. Caminada, M.: Comparing two unique extension semantics for formal argumentation: Ideal and eager. In: Proceedings of the 19th Benelux Conference on Artificial Intelligence (BNAIC 2007). (2007) 81-87

8. Dvorak, W., Gaggl, S.A.: Incorporating stage semantics in the SCC-recursive schema for argumentation semantics. In: Proceedings of the 14th International Workshop of Non-Monotonic Reasoning (NMR 2012), Rome, Italy (June 2012)

9. Gratie, C., Florea, A.M.: SCC-recursiveness revisited. In: 9th International Workshop on Argumentation in Multi-Agent Systems (ArgMAS 2012). (2012) 